\title{
Role of hypothalamic MAPK/ERK signaling in diabetes remission induced by the central action of fibroblast growth factor 1
}

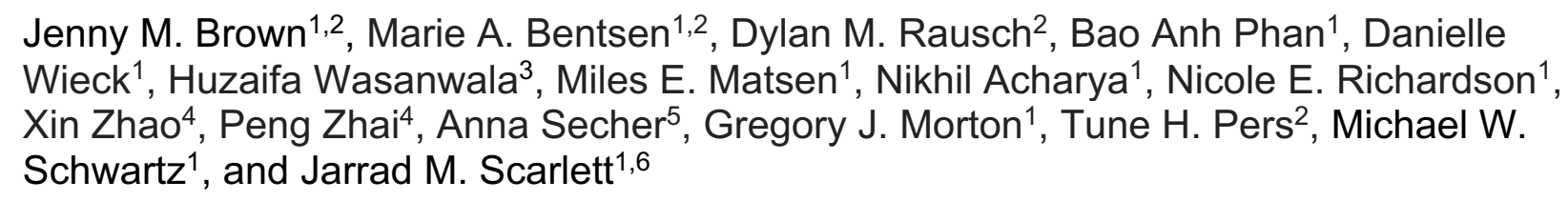

${ }^{1}$ University of Washington Medicine Diabetes Institute, Department of Medicine,

14 Seattle, WA, USA.

152 University of Copenhagen, Novo Nordisk Foundation Center for Basic Metabolic

16 Research, Copenhagen, Denmark.

173 University of Central Florida, Department of Medicine, Orlando, FL, USA.

$18{ }^{4}$ Global Drug Discovery, Novo Nordisk Research China, Beijing, China.

$19{ }^{5}$ Global Drug Discovery, Novo Nordisk A/S, Maaloev, Denmark.

$20{ }^{6}$ Department of Pediatric Gastroenterology and Hepatology, Seattle Children's Hospital,

21 Seattle, WA, USA.

Address for Correspondence:

30 Jarrad M. Scarlett

$31 \quad$ Jarrad.Scarlett@seattlechildrens.org

32 University of Washington Medicine Diabetes Institute, Department of Medicine

33750 Republican St, F770

34 Seattle, Washington, 98195, USA.

35 Phone: (206) 8975282 


\section{Summary}

37 The capacity of the brain to elicit sustained remission of hyperglycemia in rodent models

38 of type 2 diabetes following intracerebroventricular (icv) injection of fibroblast growth

39 factor 1 (FGF1) is well established. Here, we show that following icv FGF1 injection,

40 hypothalamic signaling by extracellular signal-regulated kinases 1 and 2 (ERK1/2),

41 members of the mitogen-activated protein kinase (MAPK) family is induced for at

42 least $24 \mathrm{~h}$. Further, we show that in diabetic Lep ${ }^{o b / o b}$ mice, this prolonged response is

43 required for the sustained antidiabetic action of FGF1, since it is abolished by sustained

44 (but not acute) pharmacologic blockade of hypothalamic MAPK/ERK signaling. We also

45 demonstrate that FGF1 R50E, a FGF1 mutant that activates FGF receptors but induces

46 only transient hypothalamic MAPK/ERK signaling, fails to mimic the sustained glucose

47 lowering induced by FGF1. These data identify sustained activation of hypothalamic

48 MAPK/ERK signaling as playing an essential role in the mechanism underlying diabetes

49 remission induced by icv FGF1 administration.

57 Keywords: Fibroblast growth factor 1, diabetes, brain, hypothalamus, mitogen-activated 58 protein kinase, extracellular signal-regulated kinase 


\section{Introduction}

60 Fibroblast growth factor 1 (FGF1) is a prototypical FGF that, in addition to its role

61 in biologic functions ranging from brain development to angiogenesis and wound

62 repair, is implicated the in regulation of feeding and glucose homeostasis ${ }^{1,2}$.

63 Recently, FGF1 emerged as a potential antidiabetic agent that following a single

64 intracerebroventricular (icv) injection, induces remission of diabetic hyperglycemia

65 lasting weeks or months in both mouse (Lep ${ }^{o b / o b}$ and LepR $\left.{ }^{d b / d b}\right)$ and rat (Zucker

66 Diabetic Fatty (ZDF)) models of type-2 diabetes (T2D) ${ }^{3-6}$. Since the effect of icv

67 FGF1 administration to normalize diabetic hyperglycemia in a sustained manner is

68 recapitulated by microinjection of a lower dose of FGF1 directly into mediobasal

69 hypothalamus $(\mathrm{MBH})$, this brain area is implicated as a key target for this sustained

70 antidiabetic response ${ }^{5}$. While recent work points to a role for glia-neuron

71 interactions leading to increased melanocortin signaling ${ }^{7}$, as well as to FGF1-

72 induced changes in $\mathrm{MBH}$ extracellular matrix ${ }^{8}$, the signal transduction mechanisms

73 by which FGF1 action in the MBH induces sustained remission of diabetic

74 hyperglycemia is unknown.

Among various intracellular signaling cascades activated by binding of FGF1 to

76 FGF receptors (FGFRs) is the extracellular-signal-regulated kinase (ERK) pathway, a

77 major signaling cassette of the mitogen activated protein kinase (MAPK) signal

78 transduction system that communicates signals arising from activation of cell surface

79 receptors to changes of gene expression occurring in the cell nucleus ${ }^{9}$. Previous work

80 demonstrates that MAPK/ERK signaling is rapidly induced in the hypothalamus of

81 diabetic mice by FGF19, a member of the endocrine FGF family, and that this ERK 
82 activation is required for its transient glucose-lowering action ${ }^{10}$. Activation of ERK

83 signaling in the $\mathrm{MBH}$ has also been documented following icv administration of FGF1

84 and is associated with robust transcriptional changes related to the ERK pathway in

85 both tanycytes and astrocytes ${ }^{5,7}$. However, neither the role played by ERK signaling

86 in these responses nor the extent to which they contribute to the sustained

87 antidiabetic effect of icv FGF1 is known. The current work was undertaken to

88 address these questions.

We report that in diabetic Lep ${ }^{o b / o b}$ mice, the sustained antidiabetic effect of icv

90 FGF1 is abolished by pharmacologic blockade of hypothalamic MAPK/ERK

91 signaling (achieved by central administration of the MAPK inhibitor U0126), but only if

92 this blockade lasts for the $24 \mathrm{~h}$ duration of FGF1-induced activation of MAPK/ERK

93 signaling in the hypothalamus. We further report that icv injection of FGF1 R50E, a

94 FGF1 mutant that activates FGFRs and induces transient, but not sustained MAPK/ERK

95 signaling ${ }^{11,12}$, fails to mimic the sustained diabetes remission induced by icv FGF1

96 administration. We also report transcriptomic evidence pointing to a role for astrocytes

97 (and possibly tanycytes) as primary targets for the hypothalamic response to FGF1.

98 Collectively, these data indicate that durable induction of hypothalamic MAPK/ERK

99 signaling is required for sustained remission of diabetic hyperglycemia induced by

100 icv FGF1 administration and adds to growing evidence of a role for glia-neuron

101 interaction in this response.

102

103 Results 
104 Central Administration of FGF1 Induces Sustained ERK1/2 Signaling in the

105 Hypothalamus

106 Consistent with our previous observations in ZDF rats ${ }^{5}$, we found using

107 quantitative western blot that in overnight-fasted wild-type (WT) mice,

108 phosphorylation of ERK1/2 (pERK1/2, a biomarker of ERK1/2 activation) was

109 detectable in the hypothalamus within 20 min of icv injection of FGF1 (3 $\mu$ g), but not

110 following icv vehicle injection (Figure 1A). Since the duration of ERK1/2 activation is

111 a determinant of gene expression and other cellular processes engaged by ERK

112 signaling ${ }^{13}$, we next measured the time course of ERK1/2 activation in the

113 hypothalamus following a single icv injection of FGF1. We found that in WT mice,

114 activation of ERK1/2 was readily detected at both the $5 \mathrm{~h}$ (Figure 1B) and 24h

115 (Figure 1C) time points following icv FGF1 injection, but this response was no

116 longer evident at 48h (Figure 1D). In contrast, changes of hypothalamic pERK1/2

117 content were not observed at any time point after icv vehicle. Interestingly, the

118 magnitude of FGF1-induction of ERK1/2 increased over time, being nearly 3-fold

119 greater at $24 \mathrm{~h}$ than at $20 \mathrm{~min}$.

120 Next, we sought to compare the degree to which hypothalamic ERK1/2

121 signaling is induced following icv injection of FGF1 to that induced by icv FGF19, an

122 endocrine member of the FGF family that transiently improves systemic glucose

123 metabolism in diabetic rodents ${ }^{10,14-17}$. Prior work has shown that icv FGF19 induces

124 ERK1/2 signaling in the hypothalamus and that improved glucose-tolerance in

125 response to icv FGF19 requires functional ERK1/2 signaling ${ }^{10}$. Acutely, both FGF1

126 and FGF19 rapidly induce activation of ERK1/2 signaling in the $\mathrm{MBH}$ following icv 
127 injection ${ }^{5,10}$, however this effect persisted for 24 h only after treatment with FGF1

128 (Figure 1E). Therefore, whereas both FGF1 and FGF19 rapidly activate ERK1/2

129 signaling in the hypothalamus following icv delivery, only FGF1 does so in a manner

130 that is sustained for at least $24 \mathrm{~h}$.

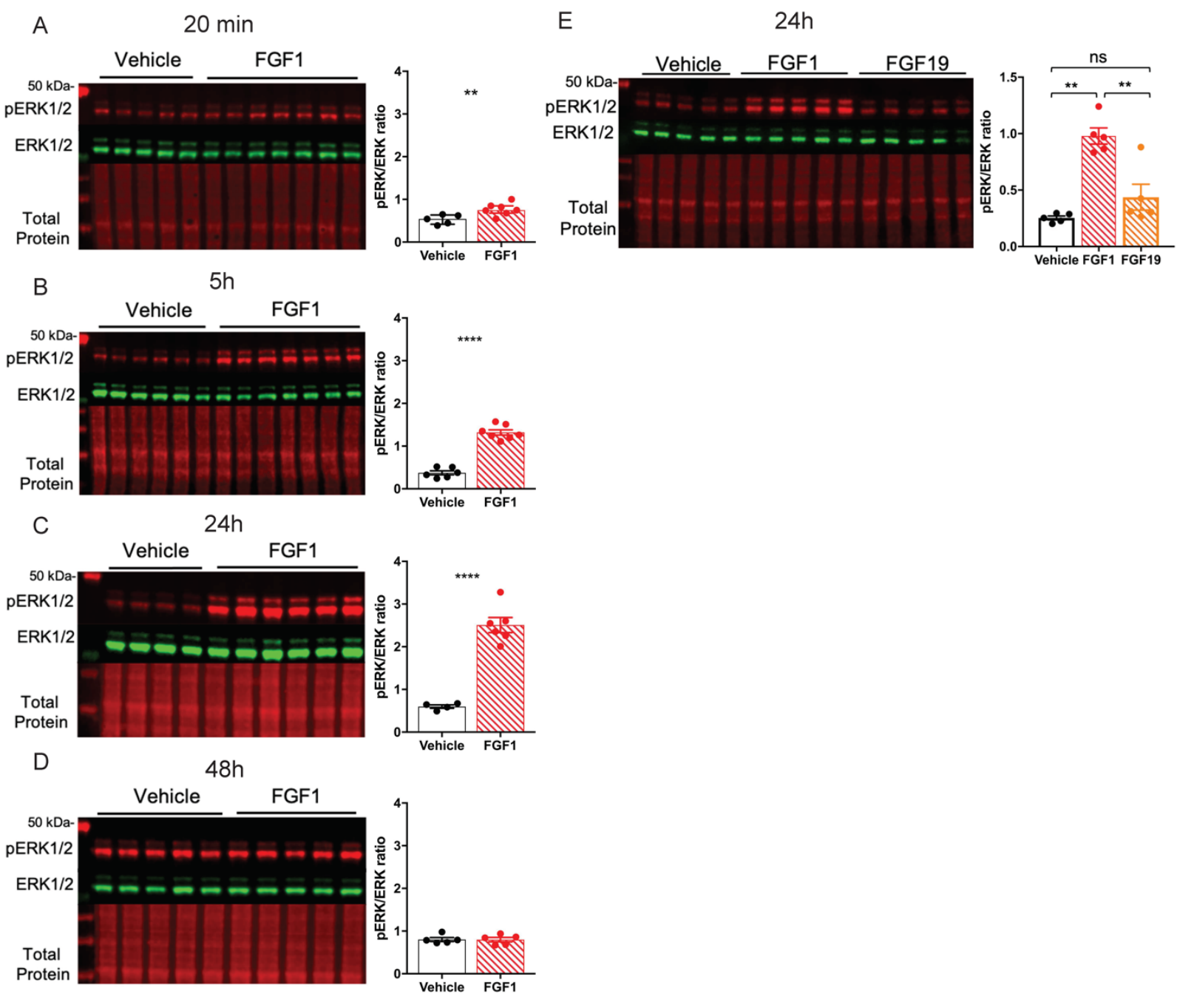

Figure 1- Central Administration of FGF1 Induces Sustained ERK1/2 Signaling in the Hypothalamus. Representative western blot (left panel) and quantitative comparison (right panel) of phosphorylated (red) and total ERK1/2 (green) and total protein (red) from hypothalamic punches from adult male C57BI6J mice after a single icv injection of either vehicle or FGF1 $(3 \mu \mathrm{g})$ at A) 20 minutes $(n=5-7 /$ group, $t=3.2497 \mathrm{df}=9.8554 \mathrm{p}=0.00444)$ B) 5 hours $(\mathrm{n}=6-7 /$ group, $t=11.937 d f=10.624 p=8.62 e-08)$ C) 24 hours ( $n=4-6 /$ group, $t=10.528, d f=5.4845, p=3.816 e-$ $05)$ and $D) 48$ hours ( $n=5 /$ group, $t=-0.0344, d f=7.8827, p=0.5133)$ after injection. $p E R K 1 / 2$ ratio unpaired Welch two sample t-test (one sided). $\left.{ }^{* *} \mathrm{P}<0.01,{ }^{* * * *} \mathrm{P}<0.0001 \mathrm{E}\right)$ Quantitative western 
blot of ERK1/2 phosphorylation 24h after a single icv injection of vehicle, FGF1, or FGF19 ( $\mathrm{n}=4$ 5/group one-way ANOVA $F(2,3.46)=54.83 p=0.00241)$.

132 Diabetes Remission Induced by Central FGF1 Delivery Requires Functional

133 Hypothalamic ERK1/2 Signaling.

134 To determine whether sustained diabetes remission induced by icv FGF1 in 135 diabetic Lep ${ }^{o b / o b}$ mice requires intact hypothalamic MAPK/ERK signaling, we co136 administered the MAPK inhibitor U0126 with FGF1 and measured its impact on 137 both hypothalamic ERK1/2 induction and on the duration of glucose lowering. We 138 found that whereas activation of hypothalamic ERK1/2 signaling following icv 139 administration of FGF1 (3 $\mu \mathrm{g})$ was acutely blocked by pre-treatment with a single 140 icv injection of U0126 (5 $\mathrm{gg})$ (Figure 2A), this intervention did not prevent FGF1-

141 induced reductions of food intake or body weight, nor did it block sustained lowering 142 of the blood glucose level (Figures 2B-D). To explain this finding, we examined the 143 duration of the effect of icv injection of $U 0126(5 \mu \mathrm{g})$ on induction of hypothalamic 144 ERK1/2 by icv administration of FGF1 $(3 \mu \mathrm{g})$. We found that although this U0126 145 pre-treatment regimen was effective early on, it did not attenuate FGF1-induced 146 activation of hypothalamic ERK1/2 at the $24 \mathrm{~h}$ time point (Figure S1A).

147 This observation prompted us to identify a route, dose, and duration of icv 148 U0126 treatment capable of blocking FGF1-induced ERK1/2 activation for a full 24h 149 period. Whereas neither repeated icv injection of U0126 (Figure S1B) nor 150 continuous infusion of U0126 into the lateral ventricle (Figure S1C) were effective, 151 we asked whether administration into the $3^{\text {rd }}$ ventricle would be more effective, 152 owing to its close proximity to the $\mathrm{MBH}$. Indeed, we found that continuous infusion 
153 of U0126 into the $3^{\text {rd }}$ ventricle for $24 \mathrm{~h}$ (by osmotic mini-pump) following a single

154 intra-3 ${ }^{\text {rd }}$ ventricular U0126 injection was sufficient to block hypothalamic FGF1-

155 induced ERK1/2 activation for a full 24h (Figure S1C).

156 We therefore employed this $24 \mathrm{~h} 3^{\text {rd }}$ ventricular U0126 administration protocol

157 to investigate the role played by MBH MAPK/ERK1/2 signaling in metabolic

158 responses elicited by icv FGF1 injection (Figure 2E) in diabetic Lep ${ }^{o b / o b}$ mice. We

159 found that whereas this intervention did not prevent initial reductions of food intake,

160 body weight, or blood glucose induced by icv injection of FGF1 (3 $\mu$ g) (Figures 2F-

$161 \mathrm{H}$ ), it did prevent sustained remission of diabetic hyperglycemia (Figure $2 \mathrm{H}$ ).

162 Because our previous work has shown that the initial glucose-lowering effect of icv

163 FGF1 injection is driven primarily by transient anorexia ${ }^{3,4}$, a group of vehicle-treated

164 Lep ${ }^{o b / o b}$ mice that were pair-fed to the intake of the group receiving FGF1 was

165 included in this study. Data from this group demonstrates that in animals receiving

166 icv vehicle treatment, pair-feeding to match the reduced food intake induced by icv

167 FGF1 injection was sufficient to lower blood glucose levels initially. However, this

168 glucose-lowering effect was not sustained irrespective of whether pair-feeding was

169 accompanied by icv U0126 or vehicle administration (Figure 2E). Nevertheless, in

170 mice that received both icv FGF1 injection and the $24 \mathrm{~h}$ U0126 infusion, blood

171 glucose levels returned to their elevated baseline value even before food intake and

172 body weight had done so, whereas glucose levels remained low throughout the

173 duration of the study in mice receiving FGF1 coupled with vehicle instead of U0126

174 (Figures 2E-H). Taken together, these results show that while hypothalamic

175 ERK1/2 activation is not required for the acute, transient effects of icv FGF1 on 
176 levels of blood glucose, food intake, or body weight, it is required for sustained

177 remission of diabetic hyperglycemia induced by the central action of FGF1.

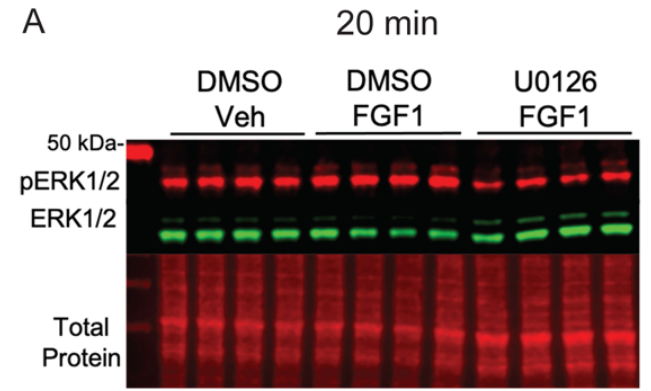

B

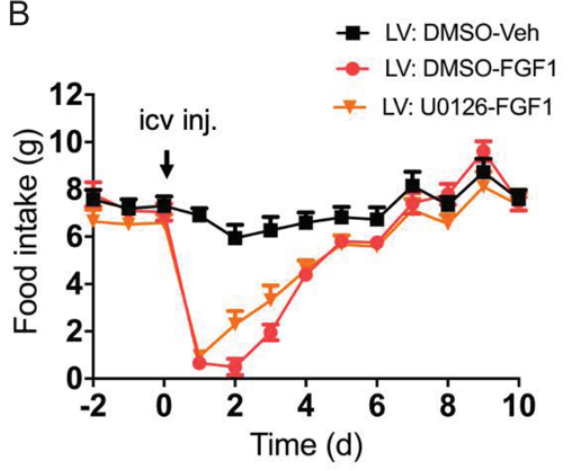

E Pre icvinj.

DMSO or Implant

U0126 Osmotic

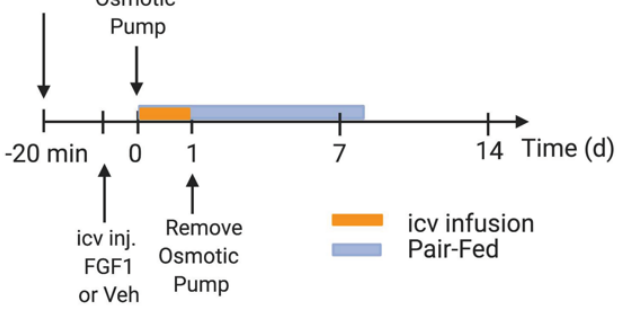

$\mathrm{F}$

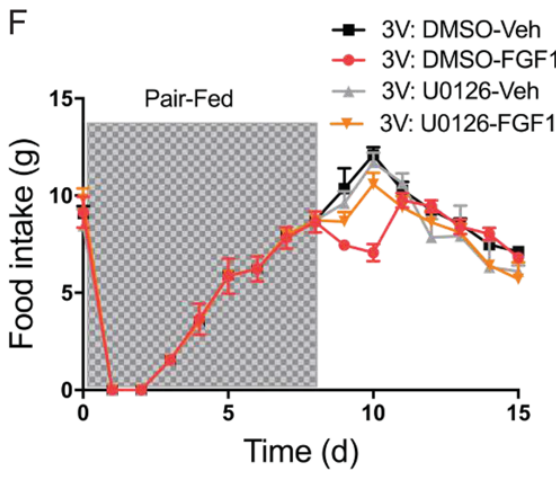

C
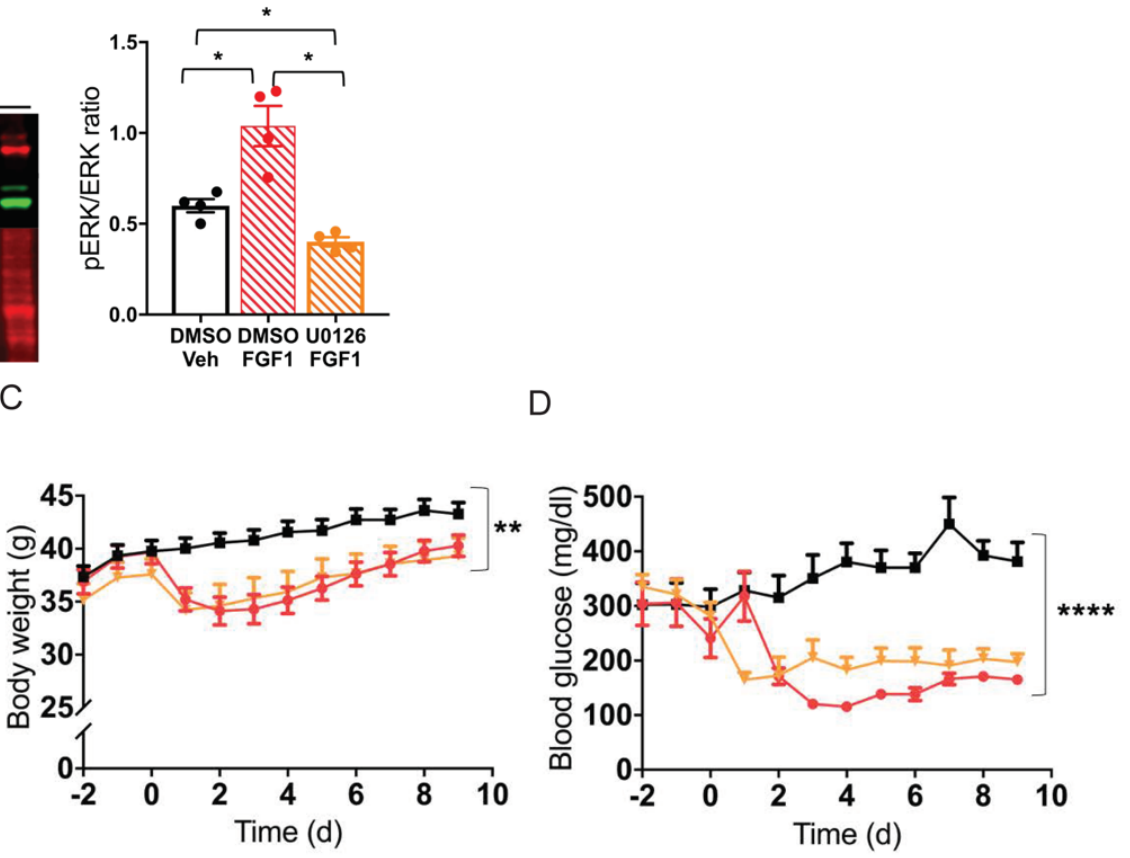

G

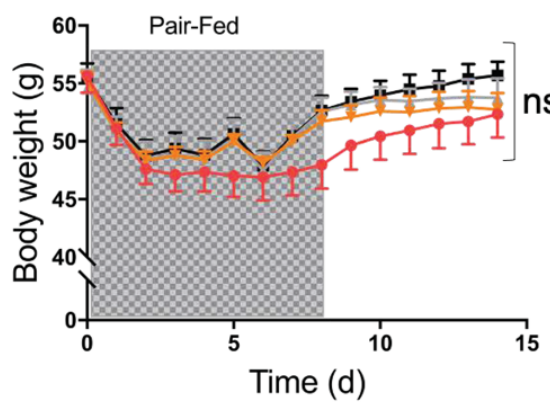

$\mathrm{H}$

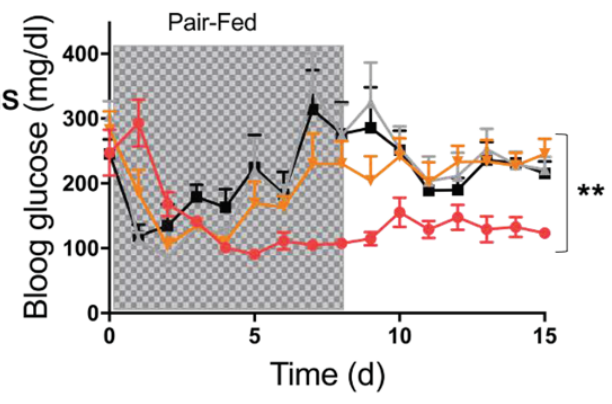

Figure 2- Prolonged MAPK signaling in the MBH is required for diabetes remission induced by central FGF1. A) Representative western blot (left panel) showing phosphorylated (red) and total ERK1/2 (green) and total protein (red), and data quantitation (right panel) from hypothalamic punches from adult male C57BI6J mice at $20 \mathrm{~min}$ after a 
injection into the lateral ventricle of the inhibitor of MAPK signaling U0126 (5 $\mu \mathrm{g})$ or DMSO, followed by injection of either vehicle or FGF1 ( $n=5 /$ group, one-way ANOVA F(2-5.27)= $20.1692 p=0.00339)$. Effects of the same treatments on levels of $\mathbf{B}$ ) food intake, $\mathbf{C}$ ) body weight ( $\mathrm{n=7-8/group;}$ repeated measures nparLD ANOVA.test statistic treatment $=4.61 \mathrm{df}=1.91$ $p=0.01)$ and $D)$ blood glucose $(n=7-8 /$ group repeated measures nparLD ANOVA.test statistic treatment $=27.96 \mathrm{df}=1.8 \mathrm{p}=7.36 \mathrm{e}-12$ ) in Lep ${ }^{o b / o b}$ mice. E) Strategy for MAPK/ERK inhibition by continuous $3 \mathrm{~V}$ infusion of U0126 or Vehicle DMSO for 24 hours followed by disconnection of osmotic pump and metabolic phenotyping image created with BioRender.com. Levels of F) food intake $\mathbf{G}$ ) Body weight ( $\mathrm{n=5-8/group} \mathrm{repeated} \mathrm{measures} \mathrm{nparLD} \mathrm{ANOVA.test} \mathrm{statistic}$ treatment $=0.80 \mathrm{df}=2.70 \mathrm{p}=0.47)$, and $\mathbf{H})$ blood glucose $(\mathrm{n}=5-8 /$ group repeated measures nparLD ANOVA.test statistic treatment $=4.99 \mathrm{df}=2.67 \mathrm{p}=0.002$ ) measured for 15 days posttreatment with icv injection of either saline vehicle or FGF1 (3ug) followed by a $24 \mathrm{~h}$ continuous infusion of U0126 or DMSO vehicle into the third ventricle.

The FGF1 Mutant FGF1 R50E Mimics the Effect of Native FGF1 to Rapidly

180 Induce ERK1/2 Activation in the Hypothalamus.

As an alternative test of the hypothesis that diabetes remission induced by

182 icv FGF1 injection requires sustained ERK1/2 activation in the hypothalamus, we 183 repeated these studies in mice using FGF1 R50E, a FGF1 mutant in which arginine

184 at the amino acid position 50 is substituted with glutamate ${ }^{11}$. In cell culture studies,

185 FGF1 R50E retains the ability to bind to and activate FGFRs and induce transient

186 activation of ERK1/2, but the effect is not sustained ${ }^{11,12,18}$. Consistent with these

187 observations, we found that when given at the same dose ( $3 \mu \mathrm{g})$, activation of

188 hypothalamic ERK1/2 by icv injection of FGF1 R50E was comparable to that

189 induced by native FGF1 at the first 3 time points (20 $\mathrm{min}$, $5 \mathrm{~h}$ and $8 \mathrm{~h}$ ), but no effect

190 was observed at either $14 \mathrm{~h}$ or $24 \mathrm{~h}$ after icv injection (Figure 3A). Therefore, FGF1

191 R50E-induced ERK1/2 activation in the hypothalamus is short-lived in comparison

192 to the more durable response elicited by native FGF1 following icv injection. 
A
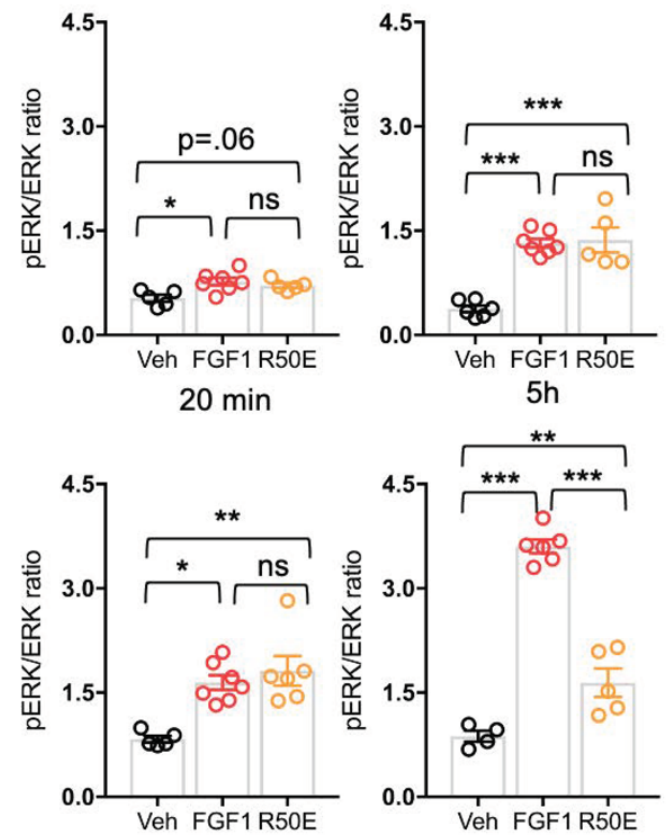

$8 \mathrm{~h}$

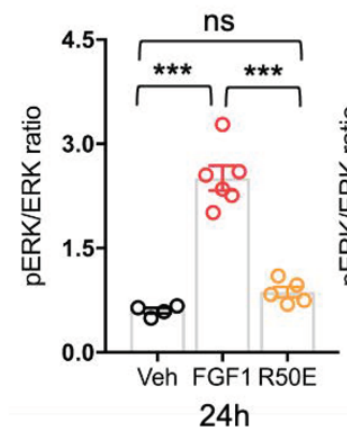

$24 \mathrm{~h}$

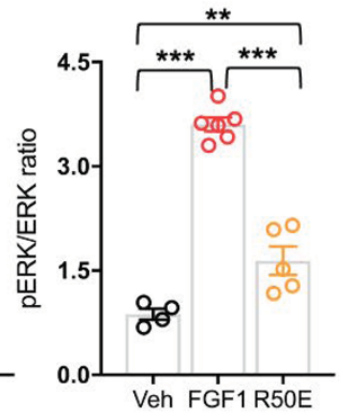

$14 \mathrm{~h}$

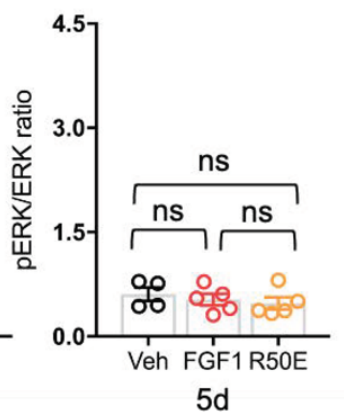

E
B

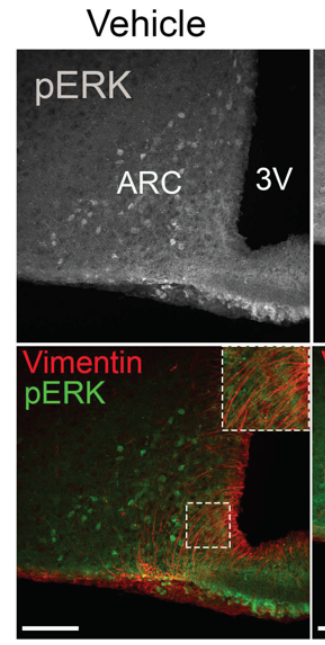

C
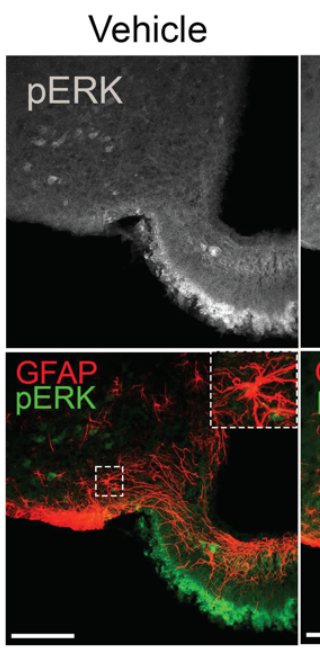

$\mathrm{F}$
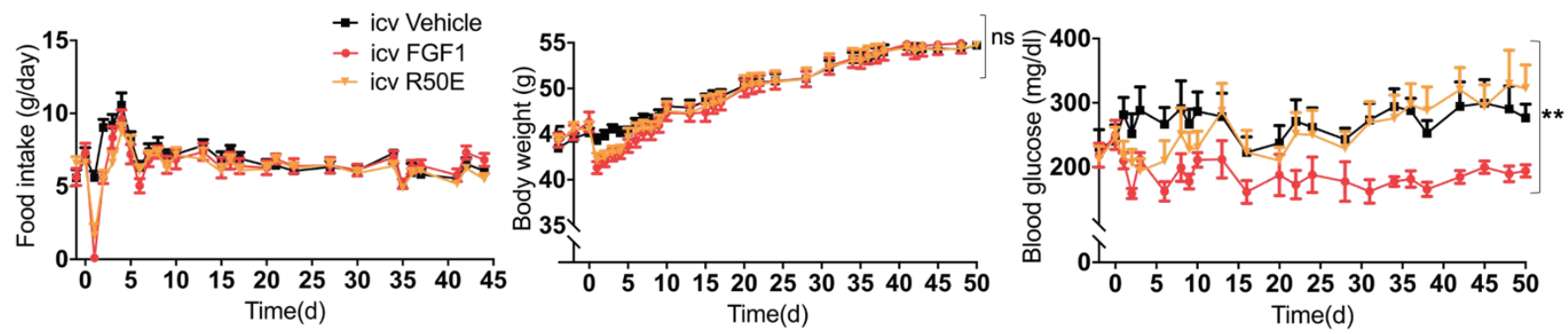

\section{Figure 3- FGF1 Mutant Induces Only Transient ERK1/2 Activation in Hypothalamus and} Fails to Induce Sustained Remission of Diabetic Hyperglycaemia. A) Quantitative western blot showing a time course of hypothalamic ERK1/2 phosphorylation after a single icv injection of either vehicle, FGF1 $(3 \mu \mathrm{g})$, or FGF1 R50E $(3 \mu \mathrm{g})$ at the following time points after injection: $20 \mathrm{~min}$ $(n=5-7 /$ group one-way ANOVA $F(2,14)=6.1616 p=0.012) ; 5 h(F(2,15)=31.334 p=4.4 e-06) 8 \mathrm{~h}$ (kruskal-wallis chi-squared $=10.361 \mathrm{df}=2 \mathrm{p}=0.0056)$; $14 \mathrm{~h}(\mathrm{n}=4$-6/group $F(2,12)=101.94 \mathrm{p}=2.95 \mathrm{e}$ 08) $24 \mathrm{~h}(\mathrm{~F}(2,12)=63.339 \mathrm{p}=4.17 \mathrm{e}-07)$; or $5 \mathrm{~d}(\mathrm{n}=4-5 /$ group $\mathrm{F}(2,11)=0.51 \mathrm{p}=0.6)$ with Tukey's post- 
hoc test ns=not significant, ${ }^{* *} P<0.01,{ }^{* * *} P<0.001,{ }^{* * *} P<0.0001$. B) confocal images of the arcuate nucleus (ARC) and third ventricle (3V) showing pERK1/2 (grey) with merged pERK1/2 (green) and vimentin (red) and C) pERK1/2 (grey) with merged pERK1/2 (green) and glial fibrillary

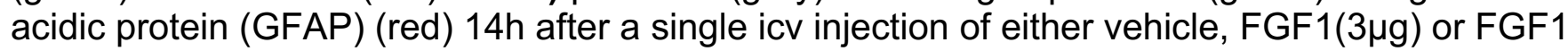
R50E $(3 \mu \mathrm{g})$. Scale bar 100 um and inserts representative double-labeled cells. Time course of the effect of the same 3 icv treatments on D) food intake, E) body weight (n=8-9/group repeated measures nparLD ANOVA.test statistic treatment=0.05 df=1.91 $p=0.94$ ), and $F$ ) plasma glucose levels ( $n=8-9 /$ group repeated measures nparLD ANOVA.test statistic treatment $=5.49 \mathrm{df}=1.79$ $\mathrm{p}=0.005)$ in Lep ${ }^{o b / o b}$ mice.

Based on previous work showing that tanycytes and astrocytes are especially

195 responsive to the effect of icv FGF1 injection to induce sustained ERK1/2

196 signaling ${ }^{5,7}$, we next examined the response of these cell types following icv

197 injection of FGF1 R50E. As anticipated, we observed that compared to icv injection

198 of native FGF1, pERK1/2 induction in both tanycytes (Figure 3B) and astrocytes

199 (Figure 3C) was markedly reduced at the 14h time point after icv injection of FGF1

200 R50E. These findings confirm that although ERK1/2 signaling is induced in

201 tanycytes and astrocytes following icv administration of either FGF1 or FGF1 R50E,

202 the response to the latter is short-lived compared to the highly durable response to

203 native FGF1.

FGF1 R50E does not Induce Sustained Remission of Diabetic Hyperglycemia.

206 Having established that unlike native FGF1, the FGF1 R50E mutant does not

207 induce sustained activation of ERK1/2 signaling in the hypothalamus following icv

208 administration, we next sought to determine whether this blunted response

209 corresponds to a reduced ability to induce sustained glucose lowering in diabetic

210 Lep ${ }^{o b / o b}$ mice. We report that in diabetic Lep ${ }^{o b / o b}$ mice, icv injection of FGF1 R50E

211 effectively recapitulated the acute reductions of food intake, body weight, and blood 
212 glucose induced by native FGF1 (Figures 3D-F). Whereas icv injection of native

213 FGF1 induced remission of diabetic hyperglycemia that was sustained for at least

$21450 \mathrm{~d}$, consistent with previous reports ${ }^{3}$, diabetic Lep ${ }^{o b / o b}$ mice treated icv with FGF1

215 R50E exhibited a complete relapse of hyperglycemia within $14 \mathrm{~d}$ of icv injection,

216 coincident with recovery of baseline food intake and body weight (Figures 3D-F).

217 Thus, whereas centrally administered FGF1 R50E mimics the acute but transient

218 effects of native FGF1 on hypothalamic ERK1/2 activation, food intake, weight loss

219 and blood glucose, neither the ERK1/2 activation nor the diabetes remission are

220 sustained in the manner seen with icv FGF1 injection. This set of outcomes

221 effectively replicates what is observed when icv FGF1 is co-administered with the

222 MAPK inhibitor U0126 for 24h, and together these data offer direct evidence that

223 the highly durable antidiabetic action of FGF1 in the brain is dependent on

224 sustained hypothalamic ERK1/2 activation.

226 Transcriptional Analysis of the Hypothalamic Response to Centrally

227 administered FGF1 and FGF1 R50E at the Single-Cell Level

$228 \quad$ To characterize transcriptional differences elicited by sustained versus

229 transient ERK1/2 activation engaged by FGF1, we leveraged a previously

230 unpublished single-cell RNA-sequencing (scRNA-seq) dataset generated as part of

231 a larger study investigating transcriptomic effects of icv injection of FGF1 in the

$232 \mathrm{MBH}$. That unpublished data was generated based on hypothalami from Lep ${ }^{o b / o b}$

233 mice icv injected with FGF1 R50E 5 days prior, and generated simultaneously with

234 the hypothalamic scRNA-seq data from the Lep ${ }^{o b / o b}$ mice icv injected with either 
235 FGF1 or vehicle also 5 days prior (Figure $4 A)^{7}$. This analysis revealed that in

236 astrocytes, tanycytes, and oligodendrocyte lineage cells, the effect of icv FGF1

237 injection to induce differentially expressed genes (DEGs; False-discovery rate,

238 FDR $<0.05$ and $\mid \log _{2}$ fold change|>0.25) was far greater than was observed following

239 icv injection of the same dose of FGF1 R50E (Figure 4B). Nevertheless, correlation

240 and ranking of differential gene expression by gene (see Methods) revealed that

241 most genes upregulated by FGF1 were also upregulated by FGF1 R50E, but at

242 much lower intensities (Figures 4C-D). From this, we infer that in the hypothalamus

243 of Lep ${ }^{o b / o b}$ mice, transient and sustained ERK activation (induced by icv injection of

244 FGF1 R50E and FGF1, respectively) induce the same gene expression programs

245 but at differing magnitudes. This interpretation is further supported by geneset

246 variation analysis of REACTOME pathways. We found that the majority of

247 'ERK/MAPK' associated pathways showed a significant change in activity,

248 specifically in astrocytes, in response to treatment (Figure 4E). Using weighted

249 gene co-expression network analysis (WGCNA) ${ }^{19}$ we further found that gene co-

250 expression modules differed significantly (linear mixed-effects models; FDR<0.05;

251 Supplementary Table 1) between FGF1 and FGF1 R50E treatment in astrocytes,

252 but not other cell types. Specifically, modules ME11 and ME13 were significantly

253 increased in astrocytes from mice receiving FGF1, but not FGF1 R50E, compared

254 to icv vehicle treatment, whereas module ME5 was significantly reduced with FGF1

255 injection, but not with FGF1 R50E. Of interest is that module M13 is significantly

256 enriched $(p=0.026)$ for genes previously reported to be induced upon astrocyte-

257 neuron interaction ${ }^{20}$. These observations support a model in which astrocyte 
258 activation resulting sustained FGF1-induced ERK1/2 signaling favors increased

259 astrocyte-neuron interaction. Whether and how such an effect might induce

260 sustained remission of diabetes is an important unanswered question.

A

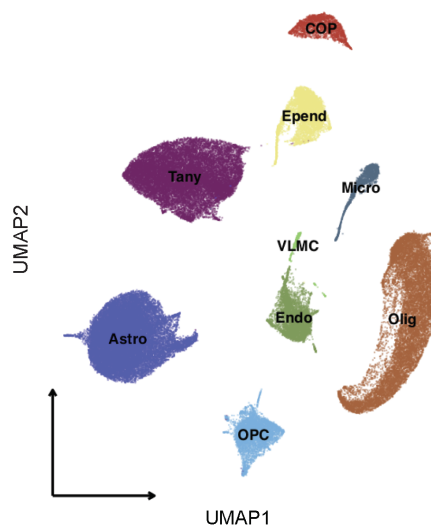

B

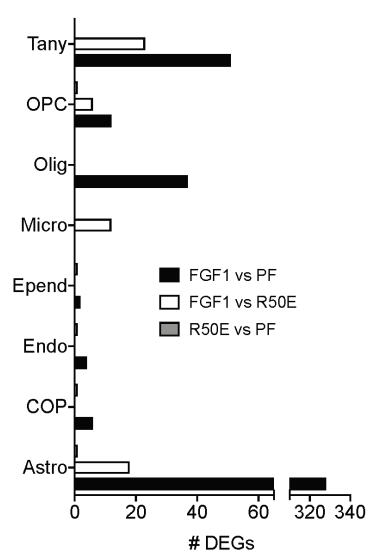

D

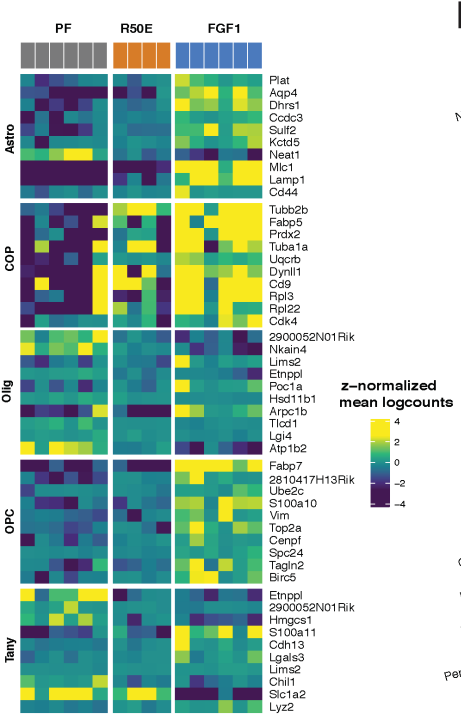

- PF $\bullet$ R50E $\bullet \mathrm{FGF} 1$

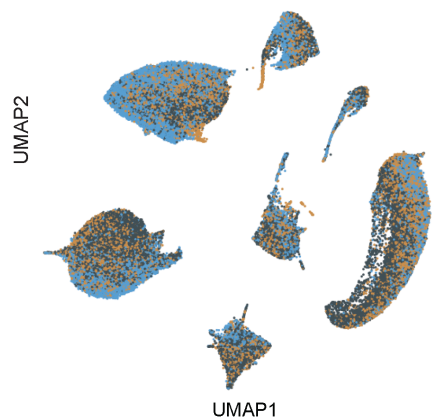

C
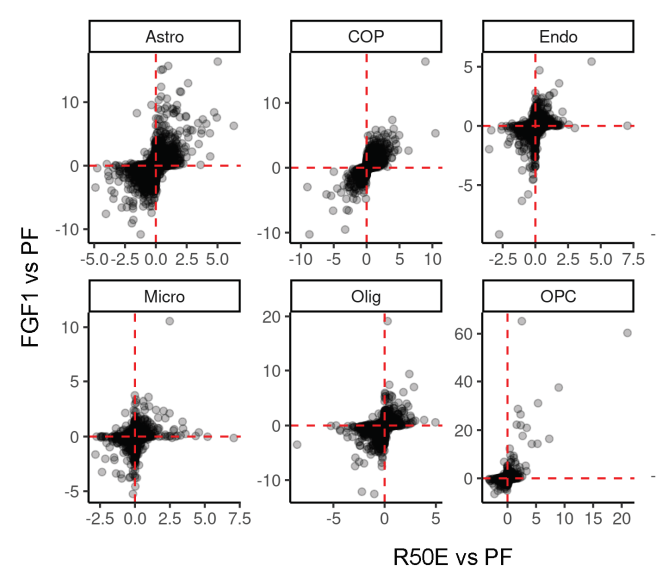

$\mathrm{E}$

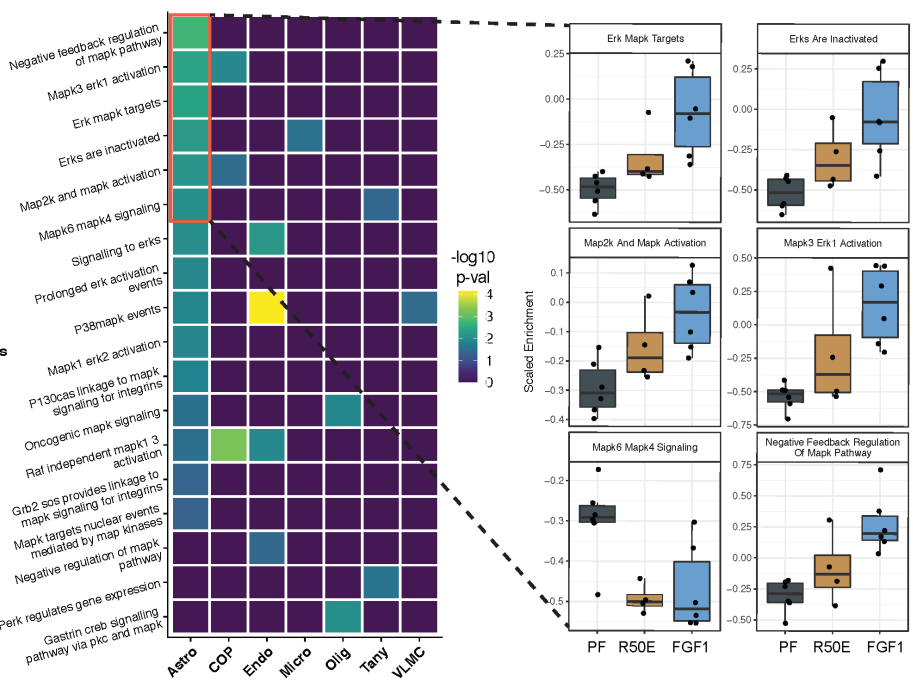


Figure 4- Hypothalamic Single-Cell Transcriptomics Reveal Transcriptional Differences between FGF1 and FGF1 R50E. A) Uniform manifold approximation and projection (UMAP) clustering of hypothalamic glia cells from diabetic Lep ${ }^{o b / o b}$ mice 5 days after a single icv injection of FGF1 or vehicle (GSE153551) or R50E. B) Number of differentially expressed genes (DEGs) identified through repeated downsampling (400 cells/group/cell type/iteration: 100 iterations). C) Correlation plot of DEGs ranked by |log2 fold change| multiplied with FDR. D) Top 10 most upregulated genes by FGF1 compared to pair-fed and FGF1 R50E in selected cell types. E) Geneset enrichment analysis of REACTOME terms related to 'MAPK/ERK' targets. Insert: scaled enrichment of top 6 enriched terms by treatment in astrocytes.

262 Discussion

In the current work, we sought to determine the role played by increased

264 hypothalamic MAPK/ERK signal transduction (measured by pERK1/2 western blot and

265 immunostaining) in the sustained antidiabetic action elicited by icv injection of FGF1 in

266 the Lep ${ }^{o b / o b}$ mouse model of T2D. Our findings demonstrate that 1) activation of

267 hypothalamic MAPK/ERK signaling is sustained for at least $24 \mathrm{~h}$ following central

268 administration of FGF1, and 2) the ability of icv FGF1 injection to induce sustained

269 normalization of diabetic hyperglycemia is dependent on this MAPK/ERK signaling

270 response, but only if it is prolonged. In contrast, hypothalamic MAPK/ERK signaling

271 does not appear to be required for transient effects on food intake, body weight, and

272 blood glucose levels induced by icv FGF1 administration. These findings identify a key

273 role for prolonged hypothalamic MAPK/ERK signaling in the sustained antidiabetic

274 action of FGF1 action in the brain.

275 MAPK/ERK pathway activation is well documented following the binding of

276 FGFRs by FGF1 ${ }^{9}$, and a role for hypothalamic ERK signaling in the regulation of

277 glucose homeostasis (in response to administration of leptin, insulin and FGF19) has

278 been reported previously ${ }^{10,21-23}$. Based on these observations, along with our recent 
279 work showing that 1) following icv FGF1 injection, robust induction of ERK1/2 signaling

280 is observed in the hypothalamic arcuate nucleus-median eminence (ARC-ME) ${ }^{5}$, and 2$)$

281 FGF1 microinjection to this brain area recapitulates sustained glucose lowering

282 observed following icv FGF1 injection ${ }^{5}$, we sought to investigate the role of

283 hypothalamic MAPK/ERK signaling in FGF1-induced sustained glucose-lowering. To

284 this end, we first characterized the time course of hypothalamic pERK1/2 induction

285 following icv injection of FGF1 at a dose known to elicit sustained glucose lowering.

286 Consistent with in vitro evidence that FGF1 induces both acute and sustained

287 activation of ERK1/2 signaling ${ }^{13}$, we report that following a single icv FGF1 injection,

288 hypothalamic pERK1/2 induction is observed within 20 min and persists for a full

$28924 h$.

290 Our next goal was to determine if this pattern of ERK1/2 activation is required

291 for the sustained antidiabetic effect of icv FGF1, and two separate approaches

292 were employed. First, we administered U0126, a potent and selective inhibitor of

293 MAPK, as a single icv injection prior to icv administration of FGF1. Although this

294 approach proved effective in terms of blocking FGF1-induced ERK1/2 activation in

295 the short-term, MAPK blockade by icv infusion of U0126 over a full 24h period was

296 required to block reversal of diabetic hyperglycemia in response to icv FGF1

297 injection. In contrast, this intervention had no impact on FGF1-induced anorexia,

298 weight loss and associated transient glucose lowering, suggesting that unlike

299 sustained glucose lowering, these responses to icv FGF1 are not dependent on

300 ERK1/2 activation. Collectively, these data suggest that the sustained antidiabetic

301 action of icv FGF1 involves mechanisms distinct from those involved in control of 
302 food intake, with the former, but not the latter, being dependent upon sustained

303 ERK1/2 signaling in the hypothalamus.

304 Our second approach to testing this hypothesis took advantage of the fact that 305 while the FGF1 R50E mutant activates FGFRs, it elicits on only transient activation of

306 ERK1/2 in cultured cells ${ }^{18}$. Thus, we predicted that the response to icv injection of the

307 FGF1 R50E mutant would mimic what is observed when the ability of icv FGF1 to

308 induce sustained MAPK/ERK signaling is blocked by central administration of U0126.

309 Indeed, we found that although FGF1 R50E induces hypothalamic ERK1/2 activation

310 following icv injection, the effect is short-lived compared to that induced by icv FGF1

311 injection. Moreover, icv injection of FGF1 R50E fully recapitulated the effects of native

312 FGF1 on food intake, body weight and associated transient amelioration of

313 hyperglycemia, but it failed to induce sustained glucose lowering. Using two

314 complementary approaches, therefore, our findings offer direct evidence that the

315 MAPK/ERK signal transduction mechanism plays a key role in the effect of FGF1 to

316 elicit sustained diabetes remission, while an unrelated signaling pathway mediates

317 FGF1's feeding effects.

318 Relevant to these observations is the mechanism engaged by FGF1-FGFR

319 interaction that leads to sustained MAPK/ERK signal transduction. We suspect a role

320 for integrins, a family of cell adhesion receptors that interact with FGFRs to modulate

321 both receptor activity and the associated intracellular response $11,18,24$. The more

322 durable and robust activation of intracellular signal transduction that results from

323 FGFR-integrin co-activation is implicated in DNA synthesis, cell proliferation, cell

324 migration, and other sustained cellular responses to growth factor signaling ${ }^{11,25}$. 
325 Integrins are also implicated in the control of insulin sensitivity in peripheral tissues in

326 skeletal muscle and liver 26,27 , but not in the regulation of glucose homeostasis by the

327 brain. As the FGF1 R50E mutant was previously characterized (based on work in cell

328 culture) as being unable to activate FGFR1 without engaging integrin signaling, we

329 favor a model in which the latter is induced in the hypothalamus by native FGF1,

330 but not FGF1 R50E, and that this effect is required for sustained ERK1/2

331 activation. Investigation into the contribution made by brain integrin signaling to the

332 antidiabetic effect of FGF1 is a priority for future work.

333 The interpretation of findings reported herein is also informed by recent work that

334 has shed light on how the hypothalamus responds to FGF1. For example, intact

335 hypothalamic melanocortin signaling (determined by interactions between Agrp and

336 Pomc neurons in the ARC) appears to be required for sustained glucose lowering

337 induced by icv FGF1 injection, but is dispensable for acute, transient effects of FGF1

338 on food intake, body weight, and glycemia ${ }^{7}$. In light of our current findings, this work

339 suggests that diabetes remission induced by icv FGF1 involves a MAPK/ERK-

340 dependent mechanism for enhanced melanocortin signaling. Whether this mechanism

341 involves direct effects of FGF1 on Agrp and/or Pomc neurons or instead is mediated

342 via an action on glial cells (e.g., tanycytes or astrocytes) warrants additional study, but

343 the associated finding that FGF1 promotes cellular contacts between astrocytes and

344 Agrp neurons supports the latter possibility ${ }^{7}$.

345 Also reminiscent of the current findings is our recent report that hypothalamic

346 perineuronal nets (PNNs, defined as extracellular matrix specializations combined of

347 proteoglycans and hyaluronan), which were recently shown to enmesh neurons in the 
348 ARC-ME ${ }^{28}$, are also targets for the glucose-lowering action of FGF1. Indeed, PNN

349 formation increases rapidly in the ARC-ME following icv FGF1 injection and, in a rat

350 model of T2D, disruption of this PNN response blocks the sustained antidiabetic action

351 induced by icv FGF1 injection, while having no impact on the acute, transient

352 effects of FGF1 on food intake, body weight, and glycemia ${ }^{8}$. These observations once

353 again parallel those observed when hypothalamic MAPK/ERK signaling either is not

354 induced or is blocked in a sustained manner. As such, they raise the interesting

355 possibility that FGF1-induced stimulation of ARC-ME PNN formation - which likely

356 involves FGF1 action on glial cells - depends upon MAPK/ERK signaling.

Additional insight into these possibilities is provided by comparing transcriptional

358 changes occurring in the hypothalamus of Lep ${ }^{o b / o b}$ mice 5 days after icv injection of

359 FGF1 vs. FGF1 R50E. While far more DEGs were induced by the former than the latter

360 peptide, a closer examination reveals that the pattern of differential gene expression is

361 in fact similar - what is different is the degree to which genes are induced. When the

362 level of gene expression against known ERK1/2-mediated pathways was interrogated

363 specifically in astrocytes, a similar pattern was detected. Namely, induction of genesets

364 in the ERK1/2 pathway was more robust following icv FGF1 than FGF1 R50E when

365 compared to pair-fed vehicle control mice, but the overall pattern of genesets was

366 similar between the two. Using a different analytic approach, we further found that a co-

367 expressed gene module detected in astrocytes following icv FGF1 injection, one that

368 was not induced by injection of FGF1 R50E, is enriched for genes that are induced

369 when astrocytes are co-cultured with neurons ${ }^{20}$. Although additional studies are needed

370 to establish the role of astrocyte-neuron interaction in the hypothalamic response to 
371 FGF1, these data support a model in which sustained ERK1/2 activation initiates a gene

372 co-expression program in astrocytes that allow them to interact with $\mathrm{MBH}$ neurons in

373 ways that profoundly impact neurocircuitry involved in controlling glucose homeostasis.

374 We conclude that sustained diabetes remission induced by icv injection of FGF1

375 involves a robust and highly durable MAPK/ERK response in the hypothalamus,

376 whereas the associated transient anorexia and loss of body weight induced by FGF1

377 does not. Although additional work is needed, convergent findings point to a role for

378 prolonged MAPK/ERK signaling in hypothalamic glial cell types as primary targets for

379 the glycemic benefit stemming from FGF1 action in the hypothalamus. Ongoing studies

380 will continue to clarify the role of glia-neuron interactions in the ability of the brain to

381 ameliorate hyperglycemia in a sustained manner in response to FGF1. 


\section{Methods}

395 Resource Availability

396 Lead Contact

397 Further information and requests for resources and reagents should be directed to

398 and will be fulfilled by the Lead Contact Jarrad M. Scarlett

399 (Jarrad.Scarlett@,seattlechildrens.org)

400

401 Materials Availability

402 This study did not generate new unique reagents

\section{Data and Code Availability}

405 The datasets generated during this study are available at NCBI Gene Expression

406 Omnibus (GEO) under Super Series number GSE153551

407 (https://www.ncbi.nlm.nih.gov/geo/query/acc.cgi?acc=GSE153551). Names, references,

408 and version numbers of all software packages used are stated in the Methods section

409 and in the Key Resource Table. Codes are available upon request.

\section{Experimental Model and Subject Details}

\section{Animals}

414 purchased from Jackson Laboratory (Bar Harbor, ME) and male, 8-week-old Lep ${ }^{o b / o b}$

415 (B6.V-Lep $\mathrm{ob} / J R j)$ were purchased from Janvier Labs, France. All animals were housed

416 individually under specific pathogen-free conditions in a temperature control 
417 environment with either a 12 h: $12 \mathrm{~h}$ or 14 h:10 h light: dark cycle, with $75-80 \%$ humidity

418 and ad libitum access to water and standard laboratory chow (LabDiet, St Louis, MO) or

419 Altromin 1310 chow (Brogaarden, Denmark). All animal procedures were performed

420 according to the National Institutes of Health Guide for the Care and Use of Laboratory

421 Animals and approved by the Institutional Animal Care and Use Committee at the

422 University of Washington or performed with approved protocols from The Danish Animal

423 Experiments Inspectorate permit number 2014-15-0201-00181 and the University of

424 Copenhagen project number P16-122.

\section{Method Details}

\section{Surgery}

429 ga, Plastics One, Roanoke, VA) were performed under isoflurane anesthesia using the 430 following stereotaxic coordinates for mice: LV: $-0.7 \mathrm{~mm}$ posterior to bregma; $1.3 \mathrm{~mm}$

431 lateral, and $1.3 \mathrm{~mm}$ below the skull surface and $3 \mathrm{~V}:-1.8 \mathrm{~mm}$ posterior to bregma; mid-

432 line and $-4.3 \mathrm{~mm}$ below the skull surface. Animals received buprenorphine

433 hydrochloride (Reckitt Benckiser Pharmaceuticals Inc., Richmond, VA) for pain relief

434 and were allowed to recover for one week prior to the study.

\section{Icv injections}

Mean blood glucose and body weight values were matched between groups

438 before the icv injections. Animals received a single icv injection via the LV of either

439 saline vehicle, recombinant mouse FGF1 (FGF1; Prospect-Tany TechnoGene Ltd., East 
440 Brunswick, NJ) dissolved in sterile water at a concentration of $1.5 \mu \mathrm{g} / \mu \mathrm{l}$, recombinant

441 human FGF1 (a generous gift from Novo Nordisk) dissolved in PBS pH 6.8, human

442 FGF1 R50E (a generous gift from Novo Nordisk) dissolved in $20 \mathrm{mM}$ Tris pH 8.0, 0.5 M

$443 \mathrm{NaCl}$ or human FGF19 (hFGF19; Phoenix Pharmaceuticals, Inc., Burlingame, CA)

444 dissolved in $0.9 \%$ normal saline at a concentration of $1.5 \mu \mathrm{g} / \mu \mathrm{l}$. and injected using a 33-

445 gauge needle (8IC315IAS5SC, 33 ga, Plastics One, Roanoke, VA) extending 0.8 mm

446 beyond the tip of the icv cannula over $60 \mathrm{~s}$ for a final volume of $2 \mu \mathrm{l}^{3}$.

447 To determine if the effect of icv FGF1 administration to induce sustained

448 remission of hyperglycemia in Lep ${ }^{o b / o b}$ mice depends on sustained MAPK/ERK

449 signaling, we studied four groups of diabetic $L e p^{o b / o b}$ mice. Two groups received a single

450 icv injection of FGF1, while the other two received icv vehicle injected into the $3 \mathrm{~V}$ using

451 a 33-gauge needle (8IC315IAS5SC, 33 ga, Plastics One, Roanoke, VA) extending 1mm

452 beyond the tip of the icv cannula. Each group also received a pre injection and an icv

453 infusion of either the selective MAPK inhibitor U0126 (662005; Millipore Sigma, St.

454 Louis, MO) dissolved in DMSO at a concentration of $30 \mathrm{mM}$ or DMSO vehicle $1 \mu \mathrm{pl}$ per

455 hour into the 3V via an micro-osmotic pump (model 1003D; Alzet, Durect Corporation,

456 Cupertino, CA) connected to the $3 \mathrm{~V}$ cannula for 24 hours.

Quantitative western blotting

Hypothalamic punches $(3 \mathrm{~mm})$ were collected after indicated treatment.

460 Dissection of a single coronal section $(1.5 \mathrm{~mm})$ between the rostral and caudal Circle of

461 Willis using an ice-cold brain matrix followed by a $3 \mathrm{~mm}$ biopunch (Harris Uni-Core, Ted

462 Pella Inc, Redding, $\mathrm{CA}$ ) of the $\mathrm{MBH}$ was flash-frozen in liquid nitrogen and stored at -80 
$463{ }^{\circ} \mathrm{C}$ until further processing. Hypothalamic punches were homogenized by sonication in

464 lysis buffer and centrifuged at $10,000 \mathrm{~g}$ for 15 minutes supernatant was collected and

465 assayed for protein concentration using a BAC assay. Lysates were mixed in the Licor

466 protein sample loading buffer and heated to $100{ }^{\circ} \mathrm{C}$ for 5 minutes. Samples were run on

467 a 10\% Bis-Tris criterion XT gel (Bio-Rad Laboratory Inc., Hercules, CA) at 200 volts for

46825 minutes electrophoretic transferred at $100 \mathrm{~V}$ for 45 minutes. Loaded protein

469 concentrations were determined based on primary antibodies 1:1000 rabbit anti-

470 pERK1/2 antibody (\#4370; Cell Signaling Technology, Danvers, MA), mouse anti-

471 ERK1/2 (\#9107; Cell Signaling Technology, Danvers, MA), secondary IRDye 680RD

472 (\#926-68073 Li-cor, Lincoln, NE), IRDye 800CW (\#92632212; Li-cor, Lincoln, NE) and

473 total protein Revert 700 Total Protein Stain Kit (\#926-11011; Li-cor, Lincoln, NE)

474 combined linear range.

475

\section{Immunofluorescence}

477 Activation of the MAPK/ERK pathway at 14 hours post icv injection pERK1/2 was

478 detected by immunohistochemistry in overnight-fasted C57Bl6J mice. Fourteen hours

479 following icv injection of either vehicle, recombinant FGF1 (3 ug), or FGF1 R50E (3 ug),

480 mice were anesthetized with ketamine and xylazine and perfused with PBS followed by

$4814 \%$ paraformaldehyde in $0.1 \mathrm{~mol} / \mathrm{L}$ PBS, after which brains were removed. Brain were

482 placed in sucrose overnight. Cryostat sectioned (30-um thick) free-floating sections

483 were permeabilized with $0.4 \%$ PBS-T overnight Wash in $0.4 \%$ PBS-T incubate in

484 pERK1/2 antibody, Host: Rabbit, (\#4370;Cell Signaling Technology, Danvers, MA)

$4851: 1000$ in (3\%BSA+0.4\%TritonX-100+ 0.2\%Normal Donkey Serum in PBS-azide) for 
24h at 4C Wash sections 5× 10min with 0.4\% PBS-T Incubate in Secondary Antibody (DaR 594-A21207) 1:1000 in (3\%BSA+0.4\%TritonX-100+ 0.2\%Normal Donkey Serum in PBS azide) for overnight in 4 C Incubate in DAPI 1:10,000 in PBS for 10min at room temperature and wash tissue $3 \times 10$ min with PBS followed by mount, dry and coverslip with PVA mounting media. Immunofluorescence images were captured using a Leica

491 SP8X Scanning Confocal microscope (Leica Microsystems, Buffalo Grove, IL) with an 492 HC FLUOTAR L 25X/0.95 W objective.

\section{Generation of single-cell suspensions}

Diabetic Lep ${ }^{o b / o b}$ mice received an icv injection of either FGF1 ( $\left.n=6\right)$, FGF1 R50E

$496(n=4)$ or vehicle $(n=6)$. Mice were euthanized 5 days later, and brains were extracted

497 between 9-12 AM, cooled in ice-cold DMEM/F12 media (Gibco, Thermo Fisher

498 Scientific) for 5 minutes. Brains were then placed ventral surface up into a chilled

499 stainless steel brain matrix and a single coronal section ( $1.5 \mathrm{~mm}$ thick) block was

500 obtained between the rostral and the caudal ends of the Circle of Willis, followed by a

501 triangular section of the MBH using a scalpel. Dissected tissue was digested using the

502 Neural Tissue Dissociation Kit from Miltenyi Biotec (Bergisch Gladbach, Germany) with

503 manual dissociation with the following modifications: sections were immediately placed

504 in preheated $\left(37^{\circ} \mathrm{C}\right)$ enzyme mix $1(1425 \mathrm{ml}$ Buffer $\mathrm{X}+37.5 \mathrm{ml}$ Enzyme $\mathrm{E})$ and

505 incubated in closed tubes for 15 minutes at $37^{\circ} \mathrm{C}$ under slow, continuous rotation. Then

506 enzyme mix 2 (15 ml Buffer $Y+7.5 \mathrm{ml}$ Enzyme A) was added followed by mechanically

507 dissociated using a wide-tipped, fire-polished Pasteur pipette by pipetting up and down

50810 times slowly. After a 10-minutes incubation under slow, continuous rotation, using a 
509 series of 2 fire-polished Pasteur pipettes with incrementally smaller openings the tissue

510 was gently dissociated until no visible pieces were observed. Cell suspensions were

511 centrifuged at $300 x g$ for 10 minutes at room temperature (RT), and resuspended in 500

512 ml Hanks' Balanced Salt Solution (HBSS) (Gibco, Thermo Fisher Scientific) and 0.04\%

$513(w / v)$ bovine serum albumin (BSA) (Sigma-Aldrich). Following an additional wash

514 (centrifugation at 300xg for 5 minutes and re-suspension in $100 \mu \mathrm{l}$ HBS $+0.04 \%$ BSA)

515 cells were filtered through a $40 \mathrm{~mm}$ mesh and $50 \mathrm{ml} \mathrm{HBSS}+0.04 \%$ BSA was added

516 through the filter and transferred to clean tubes. The cell suspension was centrifuged at

$517300 x g$ for 5 minutes and resuspended in 50-150 ml HBSS+0.04\% BSA. Cell

518 concentrations were estimated on a NucleoCounter NC-3000 (Chemometec, Denmark)

519 and kept on ice until single-cell encapsulation.

\section{Single-cell RNA sequencing}

Single-cell cDNA libraries were generated using the 10x Genomics (USA)

523 Chromium single-cell controller and the 3' v2 Reagent Kit according to manufacturer's

524 protocol. Single-cell libraries were sequenced on a NextSeq 500 platform with 3

525 samples on one flow cell to obtain 100 and 32-bp paired end reads using the following

526 read length: read 1, 26 cycles, read 2, 98 cycles and i7 index, 8 cycles.

\section{Single-cell RNA-sequencing data processing}


532 genome GRCm38 (mm10, part of the Cell Ranger software package; for the scRNA-seq

533 dataset) or an adapted reference from the mouse reference genome including introns

534 (for the snRNA-seq dataset), following the steps outlined on the 10x Genomics website

535 (https://support.10xgenomics.com/single-cell-gene

536 expression/software/pipelines/latest/advanced/references\#premrna). Barcodes were

537 filtered to include those with a total UMI count $>10 \%$ of the 99 th percentile of the

538 expected recovered cells. Next, Scrublet $^{29}$ was run with default parameters to identify

539 likely doublets which were subsequently removed from the expression matrix. Further

540 processing was performed using the Seurat ${ }^{30,31} \mathrm{R}$ package (version 2.3). Cells in which

$541<400$ or $>4,000$ expressed genes were detected, as well any cell with $>20 \%$

542 mitochondrial and/or ribosomal transcripts were discarded. Counts were normalized

543 using the SCtransform() function from Seurat. Variable genes were identified using the

544 FindVariableGenes() function with default settings. Unwanted sources of variation, e.g.

545 number of UMls and ribosomal and mitochondrial percentage, were regressed out using

546 the ScaleData() function. Principal components were identified with variable genes

547 using the RunPCA() function. The PCs for clustering were identified using the

548 maxLikGlobalDimEst function from the intrinsicDimension ${ }^{32}$ package which is described

549 as best practice ${ }^{33}$. These 10 PCs were used to identify clusters and with the

550 FindClusters() function at a resolution of 0.2 . All neuronal lineage cells were removed

551 from this dataset, as the quality was poor due to mouse age (myelination/neuron

552 fragility).

553 This dataset was then merged with the previously published part of this dataset

554 comprising of hypothalamic cells from vehicle and FGF1 treated mice. As cells merged 
555 into the expected clusters, no integration methods were required. Cluster labels from

556 the previous dataset were used to annotate the clusters found in the merged dataset.

557 The final step was to further remove any artifacts/doublets, which had been

558 missed in the initial round of processing. Each cell type underwent a round of iterative

559 clustering as described above. Within each cell type, any cluster in which $50 \%$ of cells

560 originated from a single sample was removed. The FindMarkers() function was used to

561 identify markers of each subcluster, which were removed if enriched for marker genes

562 of a different cell type.

563 Finally, for each cell, the silhouette index, a measure quantifying the similarity of

564 a given cell to its assigned cluster compared to other clusters, was computed, and

565 subsequently in order to remove cells that could not confidently be assigned to a single

566 cluster, cells with a negative silhouette index were discarded.

\section{Single-cell RNA-sequencing analyses}

569 Cell Type Abundance

570 Cell type abundance per individual sample was calculated by summing the total number

571 of cells within each defined cell cluster and then dividing by the total number of cells

572 collected per sample. Shifts in cell type abundance across conditions were tested using

573 a mixed effects linear model with a random effect for samples to account for the

574 hierarchical structure of the data.

575

576 Differential State analysis 
577 Using the Muscat package() pre-defined clusters were split by sample requiring at least

57810 cells per sample-cluster combination ${ }^{34}$. Raw counts were summed across each

579 gene-sample-cluster combination to generate a single column per sample-cluster. This

580 matrix was then fed directly into edgeR() for standard processing ${ }^{35}$. A gene was

581 considered differentially expressed if the adjusted local p-value (adjusted within a

582 cluster) was less than 0.05.

584 Correlation Analysis

585 Following DGE analysis, each gene was assigned a rank within each cluster treatment

586 combination based on its differential expression (-log10(p-value) ${ }^{*} \log 2$ FoldChange).

587 The transcriptomic response to the multiple treatment paradigms were then compared

588 to one another by calculating the spearman's correlation of gene rankings.

$590 \quad$ GSVA analysis

591 Gene set variation analysis was performed using the R package 'GSVA' function with

592 default parameter s(method="gsva") ${ }^{36}$. GSVA implements a non-parametric

593 unsupervised method for gene set enrichment that transforms the data from a gene by

594 sample matrix to a gene-set by sample matrix, allowing for individual sample gene-set

595 enrichment testing. As input to GSVA, we calculated the pseudobulk profiles from each

596 sample cluster combination which were then normalized with the variance stabilizing

597 transformation function from DESeq2. All gene sets from the REACTOME database

598 were tested for enrichment. The association between gene set enrichment and

599 treatment status was assessed using linear regression. A linear model with the 
600 normalized enrichment score as dependent and treatment group as independent

601 variable was constructed for each gene set. P-values were adjusted for multiple testing

602 using the FDR correction.

603

604 WGCNA and adjacency matrix calculation

605 The WGCNA package (version 1.66) (cite number 17) implemented in R was used ${ }^{37}$.

606 Pearson correlation coefficient was used for the single-cell data and signed network

607 parameter was used to compute the gene-gene adjacency matrix. Powers

608 corresponding to the top 95th percentile of network connectivity or above were

609 discarded and the lowest soft threshold power between 1 and 30 to achieve a scale-free

610 topology R-squared fit of 0.8 was selected.

612 WGCNA hierarchical clustering

613 The topological overlap matrices were converted to distance matrices, and the hclust()

614 function was used with the complete method to cluster genes hierarchically. The

615 cutreeDynamic() function was used with a deepSplit parameter of 2 and the pamStage

616 parameter set to FALSE to carve the dendrogram into modules with at least 15 genes.

617 The moduleEigengenes() function was used to compute module eigengenes, the vector

618 of cell embeddings on the first PC of each module's expression submatrix. The

619 mergeCloseModules() function was used to merge modules, using a cutHeight of $\leq 0.2$,

620 corresponding to a Pearson correlation between module eigengenes of $\geq 0.8$. The

621 module eigengenes and expression matrices were used with the signedKME() function

622 to compute gene-module Pearson correlations, or kMEs, a measure of how close each 
623 gene is to each module. Module treatment associations were tested using a linear

624 mixed-effects model with the Ime4 R package ${ }^{38}$ (function Imer()), using a random effect

625 for each sample. Significance values are FDR corrected to account for multiple testing.

627 Astrocyte Enrichment

628 To characterize WGCNA modules found enriched in FGF1 treated astrocytes

629 when compared to FGF1-R50E and vehicle treated mice, a hypergeometric enrichment

630 test was performed using markers of astrocytes in middle cerebral artery occlusion

631 (MCAO)/lipopolysaccharide (LPS) mice or astrocytes co-cultured with neurons. Prior to

632 the analysis, the (GSE35338) ${ }^{39}$ and (E-MTAB-5514) ${ }^{20}$ datasets were downloaded and

633 DEG analysis was performed. Genesets were constructed by identifying any gene with

$634{\mathrm{a} \log _{2} \text { fold-change }>2 \text { and FDR }<0.05 \text { (omitted for the GSE35338 }}^{39}$ dataset) in any of the

635 conditions described. Any genes shared between LPS and MCAO at Day 1 were

636 assigned to the PAN-reactive geneset. Furthermore, any gene found to overlap

637 between LPS and MCAO was removed from the geneset. All p-values were FDR-

638 corrected using sBH correction.

\section{Statistical Analysis}

641 Data from individual experiment are shown as dot plots representing data from

642 individual animals and bar graphs representing average \pm SEM. Statistical analyses

643 were performed using $\mathrm{R}^{40}$. Student's t-test was used to compare the means in two

644 groups and one-way ANOVA to compare three groups assumptions of test were

645 satisfied. For data that was collected repeatedly on each individual animal overtime we 
646 employed robust non-parametric methodology statistical procedures to enable accurate

647 and reliable analysis of longitudinal measurements with minimal conditions and have

648 competitive performance for small sample size when (semi)parametric assumptions

649 were are not satisfied. Longitudinal data were analyzed using a linear mixed model

650 including fixed effect of treatment and day and random effects of animal. Linear mixed

651 models were conducted with the R statistical package "nlme" 41 , or the equivalent

652 nonparametric test using the R statistical package "nparLD" 42 and followed up by post

653 hoc analysis using nparcomp ${ }^{43}$. Probability values less than 0.05 were considered

654 statistically significant.

\section{Acknowledgements}

657 The authors are grateful for the technical assistance provided Helle Kinggaard Lilja-

658 Fischer, Cecilia Ratner, Birgitte Holst, and the Single-cell Omics Platform at the Novo

659 Nordisk Foundation Center for Basic Metabolic Research, University of Copenhagen.

660 The authors thank Nathanial Peters at the University of Washington Keck Imaging

661 Center for technical assistance and the National Institutes of Health (S10-OD-016240)

662 for support to the W.M. Keck Foundation Center for Advanced Studies in Neural

663 Signaling. JMB was supported by National Heart, Lung, and Blood Institute T32 training

664 grant HL-007312 and the Diabetes Research Center Samuel and Althea Stroum

665 Endowed Graduate Fellowship and HW was supported by the NIDDK-funded Diabetes,

666 Obesity and Metabolism Training Grant (T32 DK007247) at the University of

667 Washington. This work was supported by NIH-NIDDK grants: K08DK114474 (JMS),

668 R01DK101997 (MWS), R01DK089056 (GJM), R01DK083042 (GJM and MWS) and an 
669 American Diabetes Association Innovative Basic Science Award (ADA 1-19-IBS-192;

670 GJM). This work was also supported by the NIH-NIDDK-funded Nutrition Obesity

671 Research Center (NORC; P30DK035816) and the Diabetes Research Center (DRC;

672 P30DK017047) at the University of Washington. Additional funding to support these

673 studies was provided to JMS by the UW Royalty Research Fund (RRF; A139339) and

674 to THP by the Lundbeck Foundation (Grant number R190-2014-3904). Funding was

675 also provided to MWS by Novo Nordisk (CMS-431104) and to MAB by the Novo

676 Nordisk Foundation (NNF170C0024328) and the Novo Nordisk Foundation Center for

677 Basic Metabolic Research, which is an independent research center at the University of

678 Copenhagen partially funded by an unrestricted donation from the Novo Nordisk

679 Foundation (NNF10CC1016515).

\section{Author Contributions}

$682 \mathrm{JMB}, \mathrm{MWS}$, and JMS conceived the project. JMB, MAB, THP, MWS, and JMS

683 designed the experiments. JMB, MAB, DMR, BAP, DW, HW, MEM, NER and JMS,

684 performed the experiments. JMB, MAB, DMR, BAP, XZ, PZ, AS, GJM, THP, MWS, and

685 JMS acquired, analyzed, and interpreted the data. JMB, MAB, DMR, MWS, and JMS

686 drafted and revised the manuscript. All authors approved the final version of the

687 manuscript. JMS is the guarantor of this work and, as such, had full access to all of the

688 data in the study and takes responsibility for the integrity of the data and the accuracy of

689 the data analysis. 


\section{Duality of Interest.}

693 Funding in support of these studies was provided to MWS by Novo Nordisk AS (CMS-

694 431104). No other potential conflicts of interest relevant to this article were reported.

\section{References}

1 Zakrzewska, M., Marcinkowska, E. \& Wiedlocha, A. FGF-1: From Biology Through Engineering to Potential Medical Applications. Critical Reviews in Clinical Laboratory Sciences 45, 91-135, doi:10.1080/10408360701713120 (2008).

2 Suh, J. M. et al. Endocrinization of FGF1 produces a neomorphic and potent insulin sensitizer. Nature 513, 436-439, doi:10.1038/nature13540 (2014).

3 Scarlett, J. M. et al. Central injection of fibroblast growth factor 1 induces sustained remission of diabetic hyperglycemia in rodents. Nature Medicine 22, 800-806, doi:10.1038/nm.4101 (2016).

4 Scarlett, J. M. et al. Peripheral Mechanisms Mediating the Sustained Antidiabetic Action of FGF1 in the Brain. Diabetes 68, 654, doi:10.2337/db18-0498 (2019).

5 Brown, J. M. et al. The Hypothalamic Arcuate Nucleus-Median Eminence Is a Target for Sustained Diabetes Remission Induced by Fibroblast Growth Factor 1. Diabetes 68, 1054-1061, doi:10.2337/db19-0025 (2019).

6 Tennant, K. G., Lindsley, S. R., Kirigiti, M. A., True, C. \& Kievit, P. Central and Peripheral Administration of Fibroblast Growth Factor 1 Improves Pancreatic Islet Insulin Secretion in Diabetic Mouse Models. Diabetes 68, 1462-1472, doi:10.2337/db18-1175 (2019).

7 Bentsen, M. A. et al. Transcriptomic analysis links diverse hypothalamic cell types to fibroblast growth factor 1 -induced sustained diabetes remission. Nature Communications 11, 4458, doi:10.1038/s41467-020-17720-5 (2020).

8 Alonge, K. M. et al. Hypothalamic perineuronal net assembly is required for sustained diabetes remission induced by fibroblast growth factor 1 in rats. Nat Metab, doi:10.1038/s42255-020-00275-6 (2020).

9 Raju, R. et al. A Network Map of FGF-1/FGFR Signaling System. J Signal Transduct 2014, 962962, doi:10.1155/2014/962962 (2014).

10 Marcelin, G. et al. Central action of FGF19 reduces hypothalamic AGRP/NPY neuron activity and improves glucose metabolism. Mol Metab 3, 19-28, doi:10.1016/j.molmet.2013.10.002 (2014).

11 Mori, S. et al. Direct binding of integrin alphavbeta3 to FGF1 plays a role in FGF1 signaling. J Biol Chem 283, 18066-18075, doi:10.1074/jbc.M801213200 (2008).

12 Mori, S. et al. A dominant-negative FGF1 mutant (the R50E mutant) suppresses tumorigenesis and angiogenesis. PLoS One 8, e57927, doi:10.1371/journal.pone.0057927 (2013).

13 Marshall, C. J. Specificity of receptor tyrosine kinase signaling: transient versus sustained extracellular signal-regulated kinase activation. Cell 80, 179-185, doi:10.1016/0092-8674(95)90401-8 (1995).

14 Morton, G. J. et al. FGF19 action in the brain induces insulin-independent glucose lowering. J Clin Invest 123, 4799-4808, doi:10.1172/JCI70710 (2013). 
Perry, R. J. et al. FGF1 and FGF19 reverse diabetes by suppression of the hypothalamic-pituitary-adrenal axis. Nat Commun 6, 6980, doi:10.1038/ncomms7980 (2015).

16 Lan, T. et al. FGF19, FGF21, and an FGFR1/beta-Klotho-Activating Antibody Act on the Nervous System to Regulate Body Weight and Glycemia. Cell Metab 26, 709-718 e703, doi:10.1016/j.cmet.2017.09.005 (2017).

17 Fu, L. et al. Fibroblast growth factor 19 increases metabolic rate and reverses dietary and leptin-deficient diabetes. Endocrinology 145, 2594-2603, doi:10.1210/en.2003-1671 (2004).

18 Yamaji, S. et al. A novel fibroblast growth factor-1 (FGF1) mutant that acts as an FGF antagonist. PLoS One 5, e10273, doi:10.1371/journal.pone.0010273 (2010).

19 Langfelder, P. \& Horvath, S. WGCNA: an R package for weighted correlation network analysis. BMC Bioinformatics 9, 559, doi:10.1186/1471-2105-9-559 (2008).

20 Hasel, P. et al. Neurons and neuronal activity control gene expression in astrocytes to regulate their development and metabolism. Nat Commun 8, 15132, doi:10.1038/ncomms15132 (2017).

21 Balland, E. et al. Hypothalamic tanycytes are an ERK-gated conduit for leptin into the brain. Cell Metab 19, 293-301, doi:10.1016/j.cmet.2013.12.015 (2014).

22 Mayer, C. M. \& Belsham, D. D. Insulin directly regulates NPY and AgRP gene expression via the MAPK MEK/ERK signal transduction pathway in mHypoE-46 hypothalamic neurons. Mol Cell Endocrinol 307, 99-108, doi:10.1016/j.mce.2009.02.031 (2009).

23 Zhang, J. et al. ERK1/2 mediates glucose-regulated POMC gene expression in hypothalamic neurons. J Mol Endocrinol 54, 125-135, doi:10.1530/JME-14-0330 (2015).

24 Desgrosellier, J. S. \& Cheresh, D. A. Integrins in cancer: biological implications and therapeutic opportunities. Nat Rev Cancer 10, 9-22, doi:10.1038/nrc2748 (2010).

25 Latko, M. et al. Cross-Talk between Fibroblast Growth Factor Receptors and Other Cell Surface Proteins. Cells 8, 455, doi:10.3390/cells8050455 (2019).

26 Kang, L. et al. Integrin-Linked Kinase in Muscle Is Necessary for the Development of Insulin Resistance in Diet-Induced Obese Mice. Diabetes 65, 1590-1600, doi:10.2337/db15-1434 (2016).

27 Williams, A. S. et al. Integrin-Linked Kinase Is Necessary for the Development of DietInduced Hepatic Insulin Resistance. Diabetes 66, 325-334, doi:10.2337/db16-0484 (2017).

28 Mirzadeh, Z. et al. Perineuronal net formation during the critical period for neuronal maturation in the hypothalamic arcuate nucleus. Nature Metabolism 1, 212-221, doi:10.1038/s42255-018-0029-0 (2019).

29 Wolock, S. L., Lopez, R. \& Klein, A. M. Scrublet: Computational Identification of Cell Doublets in Single-Cell Transcriptomic Data. Cell Syst 8, 281-291.e289, doi:10.1016/j.cels.2018.11.005 (2019).

30 Butler, A., Hoffman, P., Smibert, P., Papalexi, E. \& Satija, R. Integrating single-cell transcriptomic data across different conditions, technologies, and species. Nature Biotechnology 36, 411-420, doi:10.1038/nbt.4096 (2018).

31 Stuart, T. et al. Comprehensive Integration of Single-Cell Data. Cell 177, 18881902.e1821, doi:10.1016/j.cell.2019.05.031 (2019).

32 Johnsson, K., Soneson, C. \& Fontes, M. Low Bias Local Intrinsic Dimension Estimation from Expected Simplex Skewness. IEEE Transactions on Pattern Analysis and Machine Intelligence 37, 196-202, doi:10.1109/TPAMI.2014.2343220 (2015).

786 Germain, P.-L., Sonrel, A. \& Robinson, M. D. pipeComp, a general framework for the evaluation of computational pipelines, reveals performant single cell RNA-seq preprocessing tools. Genome Biology 21, 227, doi:10.1186/s13059-020-02136-7 (2020). 
34 Crowell, H. L. et al. muscat detects subpopulation-specific state transitions from multisample multi-condition single-cell transcriptomics data. Nature Communications 11, 6077, doi:10.1038/s41467-020-19894-4 (2020).

35 Robinson, M. D., McCarthy, D. J. \& Smyth, G. K. edgeR: a Bioconductor package for differential expression analysis of digital gene expression data. Bioinformatics 26, 139140, doi:10.1093/bioinformatics/btp616 (2009).

36 Hänzelmann, S., Castelo, R. \& Guinney, J. GSVA: gene set variation analysis for microarray and RNA-Seq data. BMC Bioinformatics 14, 7, doi:10.1186/1471-2105-14-7 (2013).

37 Langfelder, P. \& Horvath, S. WGCNA: an R package for weighted correlation network analysis. BMC Bioinformatics 9, 559, doi:10.1186/1471-2105-9-559 (2008).

38 Bates, D., Mächler, M., Bolker, B. \& Walker, S. Fitting Linear Mixed-Effects Models

Using Ime4. 2015 67, 48, doi:10.18637/jss.v067.i01 (2015).

80039 Zamanian, J. L. et al. Genomic Analysis of Reactive Astrogliosis. The Journal of

$801 \quad$ Neuroscience 32, 6391-6410, doi:10.1523/jneurosci.6221-11.2012 (2012).

80240 R: A Language and Environment for Statistical Computing (Vienna, Austria, 2013).

80341 Jose Pinheiro, D. B., Saikat DebRoy, Deepayan Sarkar, and R Core Team. nlme: Linear and Nonlinear Mixed Effects Models. (2013).

42 Konietschke, K. N. a. Y. R. G. a. E. B. a. F. nparLD: An R Software Package for the Nonparametric Analysis of Longitudinal Data in Factorial Experiments. Journal of Software Package for Nonparametric Multiple Comparisons and Simultaneous 